Check for updates

Cite this: RSC Adv., 2017, 7, 21380

Received 1st March 2017 Accepted 9th April 2017

DOI: $10.1039 / c 7 r a 02520 c$

rsc.li/rsc-advances

\section{A green, facile, and rapid method for microextraction and Raman detection of titanium dioxide nanoparticles from milk powder $\dagger$}

\author{
B. Zhao, (D) ${ }^{a}$ X. Cao, ${ }^{\text {a }}$ R. De La Torre-Roche, ${ }^{c}$ C. Tan, ${ }^{a}$ T. Yang, ${ }^{a}$ J. C. White, (D) \\ H. Xiao, (iD a B. Xing iD ${ }^{b}$ and L. He*a
}

Titanium dioxide $\left(\mathrm{TiO}_{2}\right)$ has been widely used as a common ingredient in numerous food products. Recently, the analysis of $\mathrm{TiO}_{2}$ nanoparticles (NPs) has attracted increasing attention due to potential risks to human health and the environment. Herein, we present a green, facile, and rapid method using flavonoid-assisted microextraction and Raman spectroscopy for $\mathrm{TiO}_{2} \mathrm{NPs}$ (anatase, $21 \mathrm{~nm}$ ) detection in real food samples. Flavonoids can bind onto $\mathrm{TiO}_{2} \mathrm{NPs}$, enabling the microextraction of the particles by ethyl acetate and sodium chloride. The extracted $\mathrm{TiO}_{2} \mathrm{NPs}$ concentrate at the interlayer and are easily removed for analysis by Raman spectroscopy. By taking advantage of surface-enhanced Raman spectroscopy (SERS), we evaluated and selected the best binding flavonoid, myricetin (MYC) bound onto $\mathrm{TiO}_{2} \mathrm{NPs}$, and were able to achieve detection at concentrations as low as $0.2 \mathrm{mg} \mathrm{L}^{-1} \mathrm{TiO}_{2} \mathrm{NPs}$ in water. The method was successfully challenged in the presence of various interferences from common food components and different $\mathrm{pH}$ conditions. The recoveries determined using inductively coupled plasma mass spectrometry (ICP-MS) ranged from $66.6 \%$ to $88.3 \%$. More important, the method showed good capability for the extraction and quantification of $\mathrm{TiO}_{2} \mathrm{NPs}$ from infant milk powder dilutions. As flavonoids, ethyl acetate, and sodium chloride have low toxicity and are relatively abundant in the environment, this may be considered a green approach for $\mathrm{TiO}_{2} \mathrm{NP}$ extraction and detection. The developed method in this study holds great potential for rapid ( $<1 \mathrm{~h}$ ) detection of $\mathrm{TiO}_{2} \mathrm{NPs}_{\mathrm{s}}$ from food products.

\section{Introduction}

In the past decades, titanium dioxide $\left(\mathrm{TiO}_{2}\right)$ has been considered an inert and safe material and has been used in many applications. Approximately 7 million tons of $\mathrm{TiO}_{2}$ are produced annually and are used as a pigment in order to provide whiteness and opacity to a variety of products. More recently, with the development of nanotechnology, $\mathrm{TiO}_{2}$ nanoparticles (NPs) with a number of unique and useful properties have been manufactured, with increasing production to 60000 tons per year by 2025. ${ }^{1}$ The presence of $\mathrm{TiO}_{2}$ NPs has been reported not only in consumer products, but also food products. Food-grade $\mathrm{TiO}_{2}$ (E171) has been widely used as a common ingredient in numerous products. Studies have shown that in E171,

\footnotetext{
${ }^{a}$ Department of Food Science, University of Massachusetts, Amherst, Massachusetts 01003, USA. E-mail: lilihe@foodsci.umass.edu; Tel: +1 4135455847

${ }^{b}$ Stockbridge School of Agriculture, University of Massachusetts, Amherst, Massachusetts 01003, USA

${ }^{c}$ Department of Analytical Chemistry, The Connecticut Agricultural Experiment Station, New Haven, Connecticut 06511, USA

$\dagger$ Electronic supplementary information (ESI) available. See DOI: 10.1039/c7ra02520c
}

approximately $36 \%$ of the $\mathrm{TiO}_{2}$ NPs have at least one dimension less than $100 \mathrm{~nm}^{2}$ Products such as candies, sweets and chewing gums are known to contain the highest amount of $\mathrm{TiO}_{2}$ NPs. Due to their antimicrobial activity, $\mathrm{TiO}_{2} \mathrm{NPs}$ have also been used in food packaging. ${ }^{3} \mathrm{TiO}_{2}$ NPs have also been used as a photocatalyst in food packaging film to preserve fruits and vegetables. ${ }^{1}$ This large scale application of $\mathrm{TiO}_{2}$ NPs in food products and other products increases the likelihood of human exposure and release into the environment. However, a number of studies have shown that $\mathrm{TiO}_{2}$ NPs may pose considerable risks to human health and the environment. ${ }^{4,5}$ Toxicological studies show that $\mathrm{TiO}_{2}$ NPs cause adverse effects via induction of oxidative stress resulting in cell damage, genotoxicity, inflammation, and immune response.$^{6-8}$ Based on the experimental evidence from animal inhalation studies, $\mathrm{TiO}_{2} \mathrm{NPs}_{\text {have }}$ recently been classified as "possibly carcinogenic to humans" by the International Agency for Research on Cancer and as occupational carcinogen by the National Institute for Occupational Safety and Health. ${ }^{4}$ Therefore, it is critically important and urgent to assess the levels of $\mathrm{TiO}_{2}$ NPs in food products and the environment.

The conventional method for analyzing $\mathrm{TiO}_{2}$ NPs in complex matrices relies on the sample digestion with concentrated acid, 
followed by quantification with inductively coupled plasma mass spectrometry (ICP-MS) or inductively coupled plasma optical emission spectrometer (ICP-OES) for elemental titanium. ${ }^{2,9,10}$ Normally, hydrofluoric acid is used for sample digestion but this presents numerous safety and environmental hazards. As a relatively new approach, single-particle ICP-MS can achieve fast size determination of $\mathrm{TiO}_{2}$ particles. ${ }^{11}$ However, it is only suitable for $20 \mathrm{~nm}$ or larger NPs, requires significant analytical expertise, involves assumptions of particle morphology, and is difficult to apply in complex or heterogeneous matrices. Majedi et al. reported on a hydrophobization and solvent microextraction method for extracting trace $\mathrm{TiO}_{2}$ from water before ICP-MS detection. ${ }^{12}$ 11-Mercaptoundecanoic acid combined with octadecylamine and cyclohexane were used as a hydrophobization reagent and extraction solvent, respectively. Although highly sensitive, these methods are still limited by several disadvantages: destruction of NPs (so the technique cannot discriminate between NPs and bulk particles), the use of toxic reagents, significant analytical expertise, and use of a complicated and time-consuming procedure (analysis time $>$ $2 \mathrm{~h}$, excluding sample digestion). Facing the need to investigate the presence of $\mathrm{TiO}_{2}$ NPs in various products and the challenge due to the large number of products, it is critically important to develop a rapid and facile method to extract and detect $\mathrm{TiO}_{2}$ NPs in various matrices.

Herein, we developed a novel approach using flavonoidassisted extraction and Raman detection for simple, green and fast analysis of $\mathrm{TiO}_{2}$ NPs. Flavonoids are naturally occurring plant polyphenolic compounds with a wide range of biological and pharmacological properties. ${ }^{13-15}$ Importantly, many flavonoids contain catechol groups, suggesting that they can act as bidentate ligands for $\mathrm{TiO}_{2}$ NPs. ${ }^{16-18}$ Furthermore, considering their hydrophobicity and ability to form charge transfer complexes with $\mathrm{TiO}_{2}{ }^{19}$ flavonoids may be a potential green ligand molecule which could be used for the extraction of hydrophilic $\mathrm{TiO}_{2} \mathrm{NPs}^{20}$ The interaction between flavonoids and $\mathrm{TiO}_{2}$ NPs can be measured and characterized using surface enhanced Raman spectroscopy (SERS) due to the charge transfer mechanism. ${ }^{\mathbf{1 6 - 1 8 , 2 1 - 2 7}}$

In this study, we first evaluated three flavonoids and selected the best binding ligand bound onto $\mathrm{TiO}_{2}$ NPs through competitive adsorption experiments with SERS. Then a flavonoid-assisted microextraction was developed, and the extracted $\mathrm{TiO}_{2}$ NPs were detected and quantified based on both of the intrinsic Raman peak of $\mathrm{TiO}_{2}$ NPs and the SERS peak of flavonoid. The developed method was challenged with various interferences and different $\mathrm{pH}$ conditions. ICP-MS was used for determination of analyte recovery. Finally, the capability of the method for the extraction and quantification of $\mathrm{TiO}_{2} \mathrm{NPs}_{\text {from }}$ infant milk powder dilutions was studied, because $\mathrm{TiO}_{2} \mathrm{NPs}$ was unexpectedly found in baby formula more recently which caused great public concerns. ${ }^{28}$ To the best of our knowledge, this is the first example of combining flavonoid-assisted microextraction with Raman spectroscopy to develop a green, simple and fast method for $\mathrm{TiO}_{2}$ NPs analysis. The development of such a method will potentially fulfill the critically important need of screening $\mathrm{TiO}_{2}$ NPs in a large variety and number of products, and greatly facilitate accurate and reliable assessment of the environmental and human health risks associated with $\mathrm{TiO}_{2}$ NPs exposure.

\section{Experimental section}

\section{Chemicals and materials}

$21 \mathrm{~nm} \mathrm{TiO}_{2}$ anatase nanopowder was purchased from SigmaAldrich (St. Louis, USA). Infant milk powder (Similac) was purchased from Super Stop \& Shop Supermarket (Hadley, USA). All flavonoids, MYC, quercetin (QUC) and luteolin (LUT) were purchased from Quality Phytochemicals LLC. All other chemicals were purchased from Sigma-Aldrich (St. Louis, USA) unless otherwise stated. All aqueous solutions were prepared with ultrapure water $(18.2 \mathrm{M} \Omega \mathrm{cm})$ from Thermo Scientific Barnstead Smart2Pure Water Purification System.

\section{Adsorption study of flavonoids on $\mathrm{TiO}_{2}$ NPs}

A $1 \mathrm{~g} \mathrm{~L}^{-1} \mathrm{TiO}_{2}$ NPs aqueous stock suspension was prepared by dispersing $\mathrm{TiO}_{2}$ NPs in ultrapure water (pH 7.0) by sonication (Branson 2800 Ultrasonic Cleaner) for $15 \mathrm{~min}$ prior to use. Before adsorption of flavonoids on $\mathrm{TiO}_{2}$ NPs, the size of $\mathrm{TiO}_{2}$ nanopowder and prepared $\mathrm{TiO}_{2}$ NPs aqueous suspension were characterized using scanning electron microscopy (SEM, Magellan 400 XHR, FEI) and Zetasizer Nano-ZS (Malvern Instruments), respectively. The $\mathrm{TiO}_{2}$ nanopowder was directly casted onto carbon conductive tape for SEM characterization and diluted $\mathrm{TiO}_{2}$ NPs aqueous suspension $\left(200 \mathrm{mg} \mathrm{L}{ }^{-1}\right)$ was used for size analysis with Zetasizer Nano-ZS. MYC, QUC and LUT were dissolved in ethanol to prepare $5 \mathrm{mM}$ stock solution. For the adsorption study of flavonoids on $\mathrm{TiO}_{2}$ NPs, the above MYC, QUC and LUT stock solution (5 mM) was mixed with an equal volume of $\mathrm{TiO}_{2}$ aqueous stock suspension, respectively. After incubation overnight at ambient temperature with gentle shaking, the mixture was centrifuged at $13300 \mathrm{rpm}$ (17 000g) for $5 \mathrm{~min}$ and the supernatant was discarded. The precipitate was redispersed with water and centrifuged at 13, $300 \mathrm{rpm}$ for $5 \mathrm{~min}$ and the supernatant was discarded. This washing step was repeated two more times to completely wash away flavonoid molecules that were not bound to $\mathrm{TiO}_{2}$ NPs. The sediment was redispersed with water and $1 \mu \mathrm{L}$ was pipetted onto a gold slide (BioGold $^{\text {TM }}$ Microarray Slides, Thermo Scientific) and air dried for SERS measurement. The gold coated slide has no SERS enhancement effect but it can provide great light reflectivity. ${ }^{29,30}$

\section{Selection of flavonoid for $\mathrm{TiO}_{2}$ NPs extraction by competitive adsorption}

Competitive adsorption experiments were used to select the flavonoid with the highest binding affinity to $\mathrm{TiO}_{2} \mathrm{NPs}$. Two of the three flavonoids (MYC and QUC, MYC and LUT, QUC and LUT) were first mixed together in a volume ratio of $1: 1$. The concentration of each flavonoid in the mixture was $2.5 \mathrm{mM}$. The above mixture was then mixed with an equal volume of $\mathrm{TiO}_{2}$ NPs aqueous stock suspension and the resulting mixture was incubated overnight with gentle shaking. The mixture was centrifuged at 13, $300 \mathrm{rpm}$ for $5 \mathrm{~min}$ and the supernatant was 
discarded. The precipitate was washed three times with water by centrifugation as described above and was then redispersed with water. One $\mu \mathrm{L}$ of redispersed sediment was pipetted onto a gold slide and air dried for SERS measurement.

\section{Optimization of MYC concentration and incubation time}

MYC was selected as the optimal flavonoid due to its strong affinity to $\mathrm{TiO}_{2}$ NPs. The concentration and incubation time were optimized to enable maximum adsorption capacity for further analyte extraction. A series of concentrations of MYC (1 $\mu \mathrm{M}, 10 \mu \mathrm{M}, 100 \mu \mathrm{M}$, and $1 \mathrm{mM}$ ) were mixed with an equal

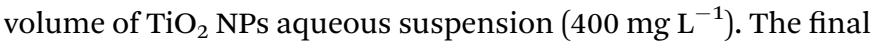
concentration of MYC in the mixture was $0.5 \mu \mathrm{M}, 5 \mu \mathrm{M}, 50 \mu \mathrm{M}$, and $500 \mu \mathrm{M}$. All the mixtures were incubated for $2 \mathrm{~h}$ at ambient temperature with gentle shaking. The resulting mixtures were then centrifuged at 13, $300 \mathrm{rpm}$ for $5 \mathrm{~min}$ and the supernatant was discarded. After washing three times with water, the precipitate was redispersed with water and $1 \mu \mathrm{L}$ was pipetted onto a gold slide and air dried for SERS measurement.

For optimization of incubation time, a $\mathrm{TiO}_{2}$ NPs suspension (400 $\mathrm{mg} \mathrm{L}^{-1}$ ) was mixed with an equal volume of MYC (1 mM in ethanol) and incubated at ambient temperature with gentle shaking. At different time points (5, 15, 30, 60 and $120 \mathrm{~min})$, an aliquot was pipetted out and centrifuged at 13, $300 \mathrm{rpm}$ for $5 \mathrm{~min}$. After washing three times with water, the precipitate was redispersed with water and $1 \mu \mathrm{L}$ was pipetted onto a gold slide and air dried for SERS measurement.

\section{SERS detection sensitivity}

SERS detection sensitivity for MYC-adsorbed $\mathrm{TiO}_{2}$ NPs was investigated to determine the overall assay performance. The optimal MYC concentration $(500 \mu \mathrm{M})$ and incubation time (30 min) were used. $\mathrm{TiO}_{2}$ NPs aqueous suspensions at various concentrations $\left(0.4,4,40\right.$, and $\left.400 \mathrm{mg} \mathrm{L}^{-1}\right)$ were mixed with an equal volume of MYC ( $1 \mathrm{mM}$ in ethanol) and were incubated for $30 \mathrm{~min}$ as described above. The resulting mixtures were then centrifuged at 13, $300 \mathrm{rpm}$ for $5 \mathrm{~min}$ and the supernatant was discarded. After washing three times with water, the precipitate was redispersed with water and $1 \mu \mathrm{L}$ was pipetted onto a gold slide and air dried for SERS measurement.

\section{Flavonoid-assisted $\mathrm{TiO}_{2} \mathrm{NPs}$ extraction from water}

Having demonstrated that MYC was the optimal flavonoid at $500 \mu \mathrm{M}$ with $30 \mathrm{~min}$ of incubation time as optimal conditions, MYC-assisted extraction of $\mathrm{TiO}_{2}$ NPs from water was evaluated. First, a series of concentrations of $\mathrm{TiO}_{2}$ NPs suspensions were mixed with an equal volume of MYC ( $1 \mathrm{mM}$ in ethanol). The final concentration of $\mathrm{TiO}_{2}$ NPs in the mixture was $0.2,2,20$, and $200 \mathrm{mg} \mathrm{L}^{-1}$. All the mixtures were incubated for $30 \mathrm{~min}$ at ambient temperature with gentle shaking. Then, the extraction was performed by mixing a $200 \mu \mathrm{L}$ aliquot of above mixture sample with $\mathrm{NaCl}$ aqueous solution ( $3 \mathrm{M}$ ) and ethyl acetate at a volume of $1: 1: 2$, respectively. After vortexing $15 \mathrm{~s}$ to facilitate phase separation, the resulting interlayer containing MYCadsorbed $\mathrm{TiO}_{2}$ NPs between the organic and aqueous phase was separated manually and $1 \mu \mathrm{L}$ was pipetted onto a gold slide and air dried for SERS measurement. To validate performance of the MYC-assisted extraction method, no NPs control and no MYC controls were also evaluated.

To assess the impact of matrix effects, $\mathrm{TiO}_{2}$ NPs suspension (800 $\mathrm{mg} \mathrm{L}^{-1}$ ) was first dispersed with an equal volume of aqueous solution containing $100 \mathrm{~g} \mathrm{~L}^{-1}$ glucose, $100 \mathrm{~g} \mathrm{~L}^{-1}$ maltose, $10 \mathrm{~g} \mathrm{~L}^{-1}$ albumin, $90 \mathrm{~g} \mathrm{~L}^{-1}$ canola oil or $100 \mathrm{~g} \mathrm{~L}^{-1} \mathrm{NaCl}$. An equal volume of MYC ( $1 \mathrm{mM}$ in ethanol) was added and the mixture was incubated for $30 \mathrm{~min}$ as described above. The final concentration of $\mathrm{TiO}_{2}$ NPs was $200 \mathrm{mg} \mathrm{L}^{-1}$. The extraction was then performed as mentioned above, followed by SERS measurement. To study the effect of $\mathrm{pH}, 400 \mathrm{mg} \mathrm{L}^{-1} \mathrm{TiO}_{2} \mathrm{NPs}$ suspensions under different $\mathrm{pH}$ conditions were prepared by dispersing $\mathrm{TiO}_{2}$ NPs in a series of aqueous solution at different $\mathrm{pH}(\mathrm{pH} 4,6,7,8$ and 10) adjusted by $\mathrm{HCl}$ or $\mathrm{NaOH}$. After adding an equal volume of MYC ( $1 \mathrm{mM}$ in ethanol), the mixture was incubated for $30 \mathrm{~min}$, followed by extraction and SERS measurement.

\section{$\mathrm{TiO}_{2}$ NPs extraction from milk powder dilutions}

A series of milk powder dispersions containing different concentrations of $\mathrm{TiO}_{2}$ NPs $\left(10,4,1,0,4\right.$ and $\left.0.1 \mathrm{~g} \mathrm{~L}^{-1}\right)$ were prepared by dispersing milk powder and $\mathrm{TiO}_{2}$ NPs with ultrapure water according to the recipe provide by manufacturer. In detail, $14 \mathrm{~g}$ milk powder and $\mathrm{TiO}_{2}$ NPs with different concentration $(1,0.4,0.1,0.04$ and $0.01 \mathrm{~g}$ ) were dispersed in $100 \mathrm{~mL}$ ultrapure water. The as-prepared $\mathrm{TiO}_{2}$ NPs-contained milk powder dispersions were then diluted 100 times with ultrapure water. $\mathrm{TiO}_{2}$ NPs extraction with MYC-assisted method was performed as described above for NPs extraction from water. The final concentration of diluted $\mathrm{TiO}_{2}$ NPs in extraction system was 50, 20, 5, 2 and $0.5 \mathrm{mg} \mathrm{L}^{-1}$, respectively. The milk powder dispersion containing $0 \mathrm{mg} \mathrm{L}{ }^{-1} \mathrm{TiO}_{2} \mathrm{NPs}$, namely no $\mathrm{TiO}_{2} \mathrm{NPS}$ control, was also tested.

\section{Raman and SERS measurements}

A DXR Raman microscope (Thermo Scientific, Madison, USA) equipped with a $780 \mathrm{~nm}$ laser and a $20 \times$ microscope objective was used in this study. All Raman and SERS spectra were obtained with a $5.0 \mathrm{~mW}$ laser power and a $50 \mu \mathrm{m}$ slit aperture for $2 \mathrm{~s}$ acquisition time. OMINC 9.0 software (Thermo Scientific) was used for Raman data acquisition and analysis. For each sample, five spots were selected randomly and scanned with the range of $100-2000 \mathrm{~cm}^{-1}$. The mean and standard deviation were analyzed. All the experiments were repeated three times.

\section{ICP-MS}

ICP-MS analysis was conducted to verify extraction efficiency of developed MYC-assisted extraction method for $\mathrm{TiO}_{2}$ NPs. Briefly, the extract containing $\mathrm{TiO}_{2}$ NPs was mixed with $500 \mu \mathrm{L}$ aqua regia (concentrated nitric and hydrochloric acids in a volume ration of $1: 3$ ) and incubated for $48 \mathrm{~h}$ at room temperature in a fume hood allowing complete digestion. After dilution to $10 \mathrm{~mL}$ with water and filtration with $0.45 \mu \mathrm{m}$ filter membrane, all the samples were analyzed with ICP-MS (Agilent 7500ce, Santa Clara, USA) to determine Ti content. 


\section{Data analysis}

All the calibration curves for quantification assays were obtained by using log 10-log 10 model:

$$
\log Y=a \log X+b
$$

where $X$ is nominal concentration of $\mathrm{TiO}_{2}$ NPs and $Y$ is Raman intensity. $a$ and $b$ were determined by linear fitting of the calibration curves.

Relative standard deviation, expressed as a percentage (\% RSD), was calculated to evaluate the precision of SERS detection:

$$
\% \operatorname{RSD}=\left(\frac{\mathrm{SD}}{\text { mean }}\right) \times 100
$$

SD and mean are the standard deviation and the mean of $\mathrm{TiO}_{2}$ NPs concentration reported by SERS detection method, respectively.

The method's accuracy was evaluated by analyzing aqueous samples and milk powder diluted samples containing different concentrations of $\mathrm{TiO}_{2}$ NPs and by calculating the percentage recovery value (\% $\mathrm{RV})$, which was defined as:

$$
\% \mathrm{RV}=\left(\frac{\left[\mathrm{TiO}_{2}\right]_{\mathrm{Cal}}}{\left[\mathrm{TiO}_{2}\right]_{\mathrm{Nom}}}\right) \times 100
$$

where $\left[\mathrm{TiO}_{2}\right]_{\mathrm{Cal}}$ is the calculated $\mathrm{TiO}_{2}$ concentration based on the calibration curves and $\left[\mathrm{TiO}_{2}\right]_{\mathrm{Nom}}$ is the actual $\mathrm{TiO}_{2}$ concentration used in the test.

The extraction efficiency was determined by calculating the percentage of extracted $\mathrm{TiO}_{2}$ concentration obtained from ICPMS analysis $\left(\left[\mathrm{TiO}_{2}\right]_{\mathrm{IM}}\right)$ of the actual $\mathrm{TiO}_{2}$ concentration added for microextraction $\left(\left[\mathrm{TiO}_{2}\right]_{\text {Nom }}\right)$ :

$$
\% \text { extraction efficiency }=\left(\frac{\left[\mathrm{TiO}_{2}\right]_{\mathrm{IM}}}{\left[\mathrm{TiO}_{2}\right]_{\mathrm{Nom}}}\right) \times 100
$$

\section{Results and discussion}

\section{Characterization of $\mathrm{TiO}_{2} \mathrm{NPs}$}

Anatase $\mathrm{TiO}_{2}$ NPs (21 nm, Sigma-Aldrich) were selected as model particles because anatase is more widely used in food and consumer products and is more toxic than rutile. ${ }^{2,8}$ We first characterized the size of both $\mathrm{TiO}_{2}$ nanopowder and aqueous suspension. The $\mathrm{TiO}_{2}$ NPs aqueous stock suspension $\left(1 \mathrm{~g} \mathrm{~L}^{-1}\right)$ was prepared by dispersing $\mathrm{TiO}_{2}$ NPs in ultrapure water $(\mathrm{pH} 7.0)$ by sonication for 15 min prior to use. As shown in Fig. S1A, $\dagger$ SEM image of $\mathrm{TiO}_{2}$ nanopowder depicts that NPs are uniform in size with an average diameter of $21 \mathrm{~nm}$, but tend to aggregate. The size distribution data of $\mathrm{TiO}_{2}$ NPs aqueous suspension shows a broad peak range from $80 \mathrm{~nm}$ to $1112.8 \mathrm{~nm}$ with an average size of $607.4 \mathrm{~nm}$ and some particles of 3-5 $\mu \mathrm{m}$ were also observed (Fig. S1B $\dagger$ ). Aggregation of the $\mathrm{TiO}_{2}$ NPs was reported to be influenced by many factors such as ionic strength, $\mathrm{pH}$, and cation valence. ${ }^{31}$ As these factors are largely varied between sample to sample in real situation, a good extraction method is expected to be able to work for all conditions regardless of their aggregation status. Therefore, in the following studies, we challenged our method with a wide range of interference.

\section{Selection of flavonoid for $\mathrm{TiO}_{2}$ NPs extraction}

Given that flavonoid-assisted extraction relies on the adsorption behavior of flavonoid molecules to $\mathrm{TiO}_{2}$ NPs, selection of an appropriate flavonoid is critical for an effective extraction method. We investigated adsorption behavior of three kinds of flavonoids; myricetin (MYC), quercetin (QUC) and luteolin (LUT) using SERS. The molecular structures of three flavonoids are shown in Fig. 1A. All SERS spectra of flavonoid-adsorbed $\mathrm{TiO}_{2}$ NPs (Fig. 1B, MYC-TiO ${ }_{2}$, QUC-TiO 2 and LUT-TiO ${ }_{2}$ ) show characteristic flavonoid peaks in the range from $800 \mathrm{~cm}^{-1}$ to $1700 \mathrm{~cm}^{-1}$ that differ from their own Raman peaks (black curves) due to charge transfer. $\mathrm{TiO}_{2}$ NPs could provide the SERS effect for adsorbed flavonoid molecule due to the charge transfer effect. The Raman signature of $\mathrm{TiO}_{2} \mathrm{NPs}$ at $144 \mathrm{~cm}^{-1}$ could be clearly observed from the SERS spectra of flavonoidbound $\mathrm{TiO}_{2}$ NPs (Fig. 1B, MYC-TiO, QUC-TiO 2 and $\mathrm{LUT}_{2} \mathrm{TiO}_{2}$ ).

Competitive adsorption studies were performed to select the

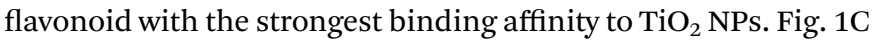
shows SERS spectra of competitive adsorption of two of three

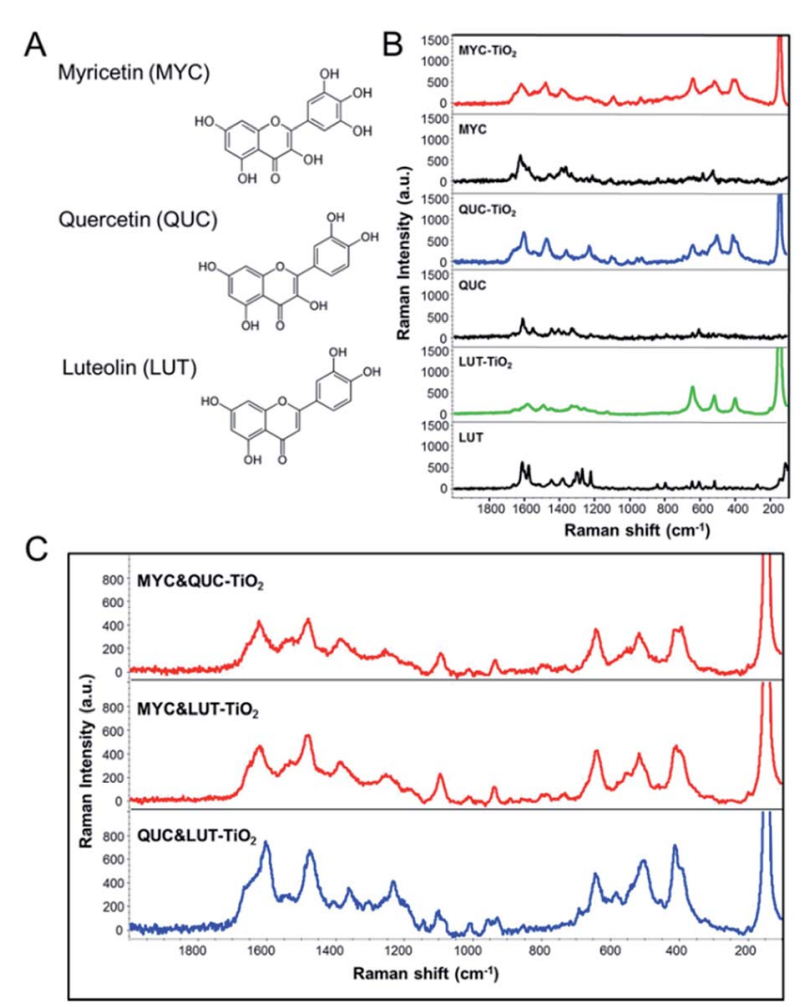

Fig. 1 (A) Molecular structure of three flavonoids: myricetin (MYC), quercetin (QUC) and luteolin (LUT). (B) Raman spectra of three flavonoids powder (MYC, QUC and LUT, black curves), and SERS spectra of MYC-adsorbed $\mathrm{TiO}_{2} \mathrm{NPs}\left(\mathrm{MYC}-\mathrm{TiO}_{2}\right.$, red curve), QUCadsorbed $\mathrm{TiO}_{2} \mathrm{NPs}$ (QUC-TiO 2 , blue curve) and $\mathrm{LUT}$-adsorbed $\mathrm{TiO}_{2}$ NPs (LUT- $-\mathrm{TiO}_{2}$, green curve). (C) SERS spectra of competitive adsorption of MYC and QUC on $\mathrm{TiO}_{2} \mathrm{NPS}$ (MYC\&QUC-TiO 2 ), MYC and LUT on $\mathrm{TiO}_{2}$ NPS $\left(\right.$ MYC\&LUT- $\mathrm{TiO}_{2}$ ), and QUC and LUT on $\mathrm{TiO}_{2} \mathrm{NPS}$ (QUC\&LUT- $\mathrm{TiO}_{2}$ ). 
flavonoids, MYC and QUC on $\mathrm{TiO}_{2}$ NPs (MYC\&QUC-TiO ${ }_{2}$ ), MYC and LUT on $\mathrm{TiO}_{2}$ NPs (MYC\&LUT-TiO ${ }_{2}$ ), and QUC and LUT on $\mathrm{TiO}_{2}$ NPs (QUC\&LUT-TiO ${ }_{2}$ ). By comparison, it is found that competitive adsorption spectra of MYC and QUC on $\mathrm{TiO}_{2} \mathrm{NPs}$ (Fig. 1C, MYC\&QUC-TiO ${ }_{2}$ ) shows the same SERS spectra as MYC-adsorbed $\mathrm{TiO}_{2}$ NPs (Fig. 1B, $\mathrm{MYC}-\mathrm{TiO}_{2}$ ), suggesting that only MYC molecules were bound to $\mathrm{TiO}_{2}$ NPs despite the presence of QUC. This indicates MYC has stronger binding affinity than QUC, so that MYC could be preferentially bind to $\mathrm{TiO}_{2} \mathrm{NPs}$ or displace QUC adsorbed on NPs. Similarly, MYC has stronger binding affinity than LUT (Fig. 1C, MYC\&LUT-TiO ${ }_{2}$ ), and QUC has stronger binding affinity than LUT (Fig. 1C, QUC\&LUT$\mathrm{TiO}_{2}$ ). Therefore, we conclude that MYC has the strongest binding affinity to $\mathrm{TiO}_{2}$ NPs and selected MYC as optimal flavonoid for following study.

\section{Optimization of MYC concentration and incubation time for sensitive SERS/Raman detection}

The concentration and incubation time were then optimized to enable maximum adsorption capacity for effective extraction and sensitive SERS/Raman detection. As shown in Fig. S2, $\dagger$ the SERS intensity increased with the increasing MYC concentration from $0.5 \mu \mathrm{M}$ to $50 \mu \mathrm{M}$; however, SERS signals reached a plateau at $50 \mu \mathrm{M}$. Response at $500 \mu \mathrm{M}$ was equivalent to that of $50 \mu \mathrm{M}$, indicating maximum adsorption of $\mathrm{MYC}$ on $\mathrm{TiO}_{2}$ NPs. Thus, $500 \mu \mathrm{M}$ was selected as the optimal concentration of MYC to ensure sufficient analyte surface adsorption. Fig. S3 $\uparrow$ shows the dependence of SERS signals on the incubation time of MYC $(500 \mu \mathrm{M})$ adsorbed on $\mathrm{TiO}_{2}$ NPs. SERS intensity increased gradually over time, and became constant at $30 \mathrm{~min}$; as such, $30 \mathrm{~min}$ was selected as the optimal incubation time for following experiments.

Different concentrations of $\mathrm{TiO}_{2}$ were incubated with MYC at the optimal concentration and incubation time to evaluate assay performance (Fig. 2). Under the Raman microscope, the aggregates of $\mathrm{TiO}_{2} \mathrm{NPs}$ dried on a gold slide were clearly seen, which facilitated the selection of effective spots for reproducible analysis. As seen in Fig. 3, the spots selected from the aggregates of $\mathrm{TiO}_{2} \mathrm{NPs}$ of high and low concentrations produced consistent signals. We also evaluated the precision of SERS detection method, which is important for the quantitative analysis of FDA-regulated products. The precision of the method was assessed by different levels $(0.2,2,20$ and $200 \mathrm{mg}$ $\mathrm{L}^{-1}$ ) of $\mathrm{TiO}_{2}$ NPs. The relative standard deviation (RSD) expressed as a percentage are reported in Table 1. All of the reported \% RSD values for our method are below 10\%, which can be considered as adequately precise according to EPA Method $6200 .^{32}$ The SERS signals increased with increasing concentration of $\mathrm{TiO}_{2}$ NPs. At concentrations as low as $0.2 \mathrm{mg}$ $\mathrm{L}^{-1}, \mathrm{TiO}_{2} \mathrm{NPs}$ could be clearly detected based on Raman peaks of both $\mathrm{MYC}$ and $\mathrm{TiO}_{2}$. $\mathrm{TiO}_{2}$ NPs could be quantified using the characteristic peak of MYC at $1615 \mathrm{~cm}^{-1}$ as indicated with the asterisk in Fig. 2A (inset). A reasonable linear response was achieved in the concentration range from $0.2 \mathrm{mg} \mathrm{L}^{-1}$ to $200 \mathrm{mg}$ $\mathrm{L}^{-1}$, with an $R$ square $\left(R^{2}\right)=0.868$. The regression equation is $\log Y=0.38 \log X+1.85$. This suggests that MYC could not only
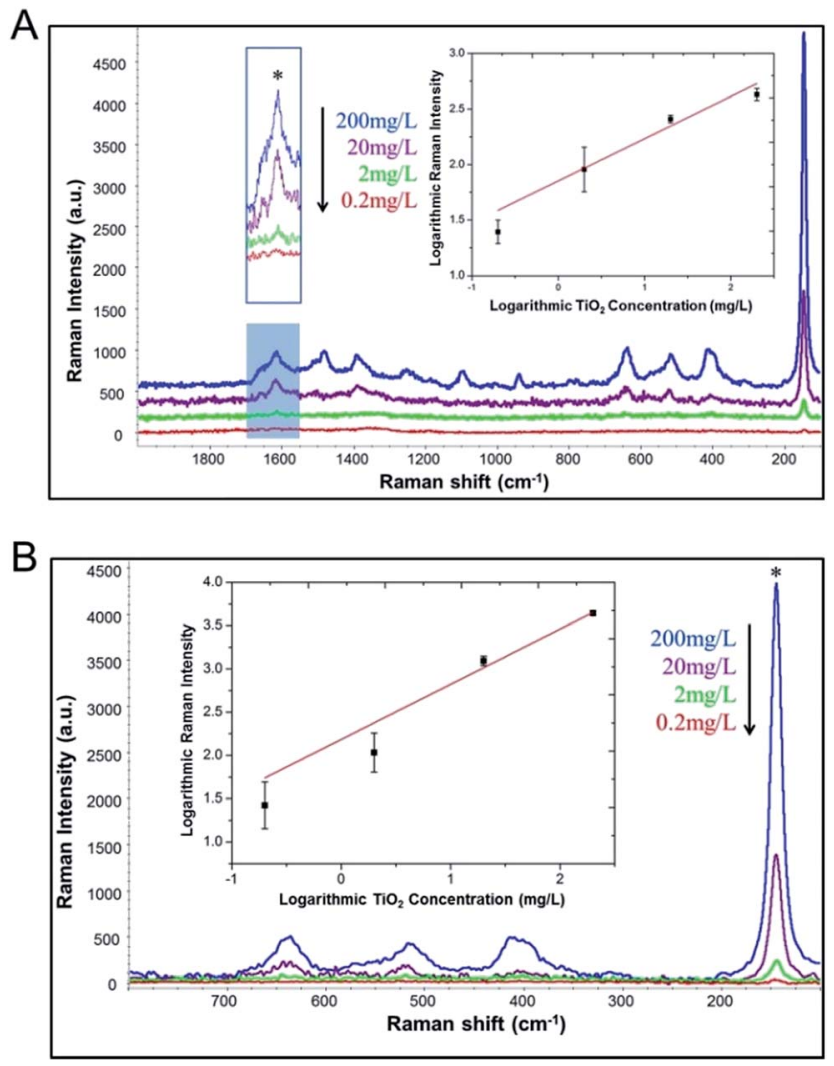

Fig. 2 (A) SERS spectra of MYC-adsorbed $\mathrm{TiO}_{2} \mathrm{NPs}$ with various concentration. Inset: linear fitting curve for $\mathrm{MYC}$-adsorbed $\mathrm{TiO}_{2} \mathrm{NPS}$ detection based on $1615 \mathrm{~cm}^{-1}$ peak of MYC indicated with asterisk. (B) Raman characteristic peaks of $\mathrm{TiO}_{2} \mathrm{NPs}$ in the range from 100-800 $\mathrm{cm}^{-1}$. Inset: linear fitting curve for quantification of $\mathrm{TiO}_{2} \mathrm{NPs}_{\text {based on }}$ $144 \mathrm{~cm}^{-1}$ Raman peak.
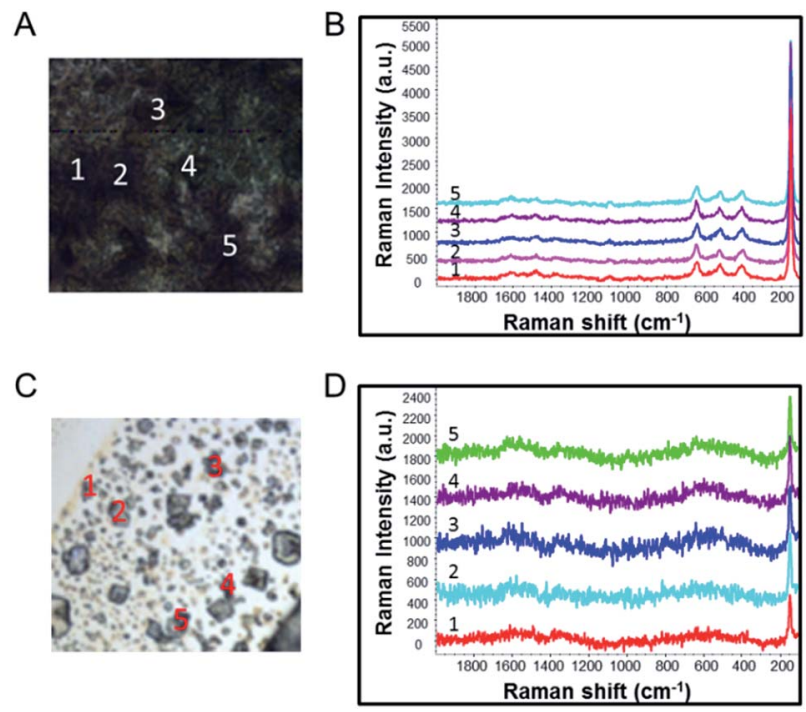

Fig. 3 (A) Photograph of $\mathrm{TiO}_{2}$ NPs sample $\left(200 \mathrm{mg} \mathrm{L}^{-1}\right)$ on gold slide. $20 \times$ microscope objective was used. (B) SERS spectra of selected positions in (A). (C) Photograph of $\mathrm{TiO}_{2} \mathrm{NPs}$ sample $\left(2 \mathrm{mg} \mathrm{L}^{-1}\right)$ on gold slide. (D) SERS spectra of selected positions in (C). 
Table 1 Evaluation of SERS detection method's precision $(n=5)$

\begin{tabular}{ll}
\hline $\begin{array}{l}\text { Nominal } \\
{\left[\mathrm{TiO}_{2}\right]\left(\mathrm{mg} \mathrm{L}^{-1}\right)}\end{array}$ & \% RSD \\
\hline 0.2 & 9.0 \\
2 & 5.8 \\
20 & 2.9 \\
200 & 0.7
\end{tabular}

be used for $\mathrm{TiO}_{2} \mathrm{NPs}$ extraction/detection, but also has potential for NPs quantification.

We also demonstrated the quantitative capability based on the intrinsic Raman peak of $\mathrm{TiO}_{2}$ itself at $144 \mathrm{~cm}^{-1}$ (Fig. 2B). In contrast, a much better linear response $\left(R^{2}=0.954\right)$ in the concentration range from $0.2 \mathrm{mg} \mathrm{L}^{-1}$ to $200 \mathrm{mg} \mathrm{L}^{-1}$ was obtained (Fig. 2B, inset). The regression equation is $\log Y=$ $0.64 \log X+2.18$. Obviously, the peak intensity of the $\mathrm{TiO}_{2}$ peaks is much greater than the MYC peaks, and as such, would be more sensitive for quantification.

\section{Extraction and Raman detection of $\mathrm{TiO}_{2}$ from water}

After optimization, a series of $\mathrm{TiO}_{2}$ NPs concentrations (0.2, 2, 20 and $200 \mathrm{mg} \mathrm{L}^{-1}$ ) were tested with our extraction method. Fig. 4A schematically illustrates the design of our flavonoidassisted microextraction method for $\mathrm{TiO}_{2}$ NPs from water. $\mathrm{TiO}_{2}$ NPs dispersed in water were first mixed with MYC solution thoroughly and then incubated to allow the binding between MYC and $\mathrm{TiO}_{2}$ NPs. Then, the microextraction was performed by mixing above mixture with $\mathrm{NaCl}$ aqueous solution (3 M) and ethyl acetate at a volume of $1: 1: 2$, respectively. The introduction of $\mathrm{NaCl}$ was to promote the extraction efficiency by decreasing the solubility of ethyl acetate in aqueous phase. After short vortex ( $15 \mathrm{~s}$ ) to facilitate phase separation, the resulting interlayer containing MYC-adsorbed $\mathrm{TiO}_{2}$ NPs between the organic and aqueous phase was separated manually and $1 \mu \mathrm{L}$ was pipetted onto a gold slide and air dried for Raman measurement. It is noteworthy that compared with conventional centrifugation method which could precipitate $\mathrm{TiO}_{2} \mathrm{NPs}$ and other components simultaneously, the use of microextraction in this study could enable the separation of $\mathrm{TiO}_{2} \mathrm{NPs}$ and eliminate the interference of other components. As shown in Fig. 4B-b, the interlayer containing MYC adsorbed $\mathrm{TiO}_{2} \mathrm{NPs}$ was clearly evident between the organic and aqueous layer after phase separation. The extraction mechanism is attributed to the surface hydrophobization of $\mathrm{TiO}_{2}$ NPs resulting from the binding between MYC molecules and $\mathrm{TiO}_{2}$ NPs through coordination interaction. ${ }^{16,17,20}$ Finally, the extracted NPs can be easily separated and detected by Raman spectroscopy. The extracted $\mathrm{TiO}_{2}$ NPs showed SERS characteristic peaks of MYC at $1615 \mathrm{~cm}^{-1}, 1480 \mathrm{~cm}^{-1}$ and $1389 \mathrm{~cm}^{-1}$, as well as Raman characteristic peaks of $\mathrm{TiO}_{2} \mathrm{NPs}$ at $636 \mathrm{~cm}^{-1}, 514 \mathrm{~cm}^{-1}, 396 \mathrm{~cm}^{-1}$ and $144 \mathrm{~cm}^{-1}$ (Fig. 4C). The SERS peaks of MYC at $1615 \mathrm{~cm}^{-1}$, $1480 \mathrm{~cm}^{-1}$ and $1389 \mathrm{~cm}^{-1}$ were assigned to $\mathrm{C}=\mathrm{O}$ stretching motion in combination with $\mathrm{C}_{2}=\mathrm{C}_{3}$ stretches, B-Ring CH inplane bending and A-Ring breath. ${ }^{18}$ To validate the efficiency of the MYC-assisted extraction method, no NPs and no MYC

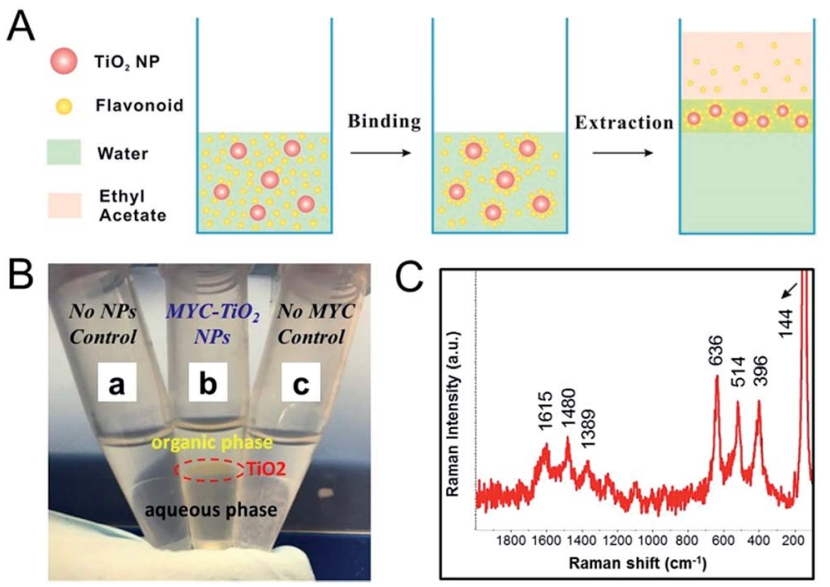

Fig. 4 (A) Schematic illustration of flavonoid-assisted microextraction method for $\mathrm{TiO}_{2}$ NPs from water. (B) Photographs of flavonoid-based phase separation. Selected flavonoid is MYC. (C) SERS spectra of MYCadsorbed $\mathrm{TiO}_{2} \mathrm{NPs}$ from the interlayer. $\mathrm{NP}=$ nanoparticle.

controls were evaluated (Fig. 4B-a and B-c). No interlayer formed in either of the controls. More importantly, in the no MYC control, all $\mathrm{TiO}_{2} \mathrm{NPs}$ were still dispersed in aqueous phase after phase separation (Fig. 4B-c) and finally precipitated to the bottom after $30 \mathrm{~min}$ (Fig. S4†), indicating that the flavonoid plays an essential role in the extraction of the NPs from water.

Fig. 5A depicts SERS spectra of different concentrations of $\mathrm{TiO}_{2}$ NPs with and without extraction. Both SERS characteristic peaks of MYC and Raman peaks of $\mathrm{TiO}_{2}$ NPs could be clearly observed. SERS spectra of extracted $\mathrm{TiO}_{2}$ NPs show comparable intensity with that of $\mathrm{TiO}_{2}$ NPs without extraction, indicating the efficacy of our method for extraction and detection of different particle concentrations. As shown in Fig. 5B, the Raman signal intensity increased as the concentration of $\mathrm{TiO}_{2}$ NPs increased from $0.2 \mathrm{mg} \mathrm{L}^{-1}$ to $200 \mathrm{mg} \mathrm{L}^{-1}$. The detection sensitivity is as low as $0.2 \mathrm{mg} \mathrm{L}^{-1}$, which is much lower than the $1 \%$ (by weight) limit set for foods. ${ }^{5}$ The extracted $\mathrm{TiO}_{2}$ NPs were quantified based on the characteristic peak of $\mathrm{TiO}_{2} \mathrm{NPs}$ at 144 $\mathrm{cm}^{-1}$ (Fig. 5B, Inset). The fitting curve shows a good linear response in the concentration range from $0.2 \mathrm{mg} \mathrm{L}^{-1}$ to $200 \mathrm{mg}$ $\mathrm{L}^{-1}$. The linear fitting equation is $\log Y=0.55 \log X+2.31$ and $R^{2}$ is 0.995 . The accuracy of the assay was evaluated by testing aqueous samples with different concentrations of $\mathrm{TiO}_{2}$ NPs and calculating percentage recovery value (\% RV) based on regression equation (Table 2). The mean $\% \mathrm{RV}$ for $\mathrm{TiO}_{2}$ assay level ranged from 83.7 to $101.6 \%$, which demonstrates that the assay is able to accurately analyze $\mathrm{TiO}_{2} \mathrm{NPs}$ in aqueous solutions.

\section{Complex food matrix effects and extraction efficiency}

Understanding the effect of various food components (e.g. sugar, protein, oil and salt) in complex food matrices to interfere the developed method is very important for practical application of the method. Therefore we challenged our method in the presence of different food components (i.e. sugar, protein, oil and salt), as well as under different $\mathrm{pH}$ conditions. 


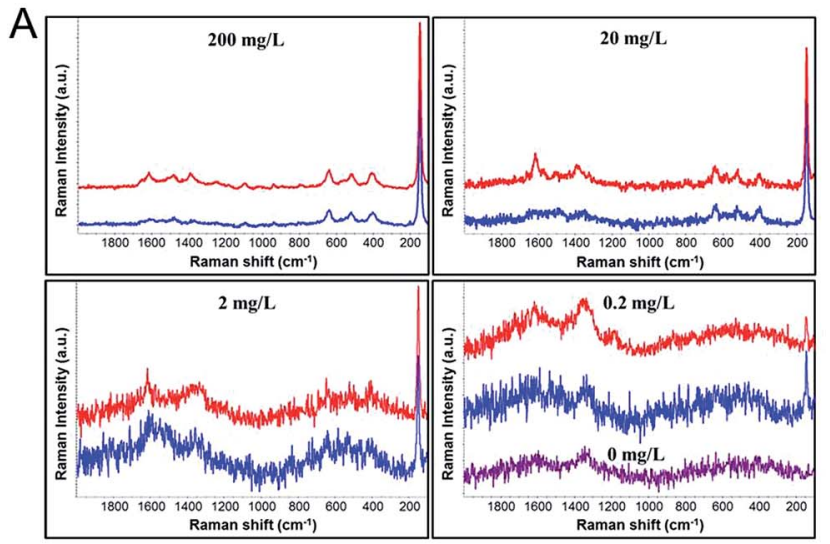

B

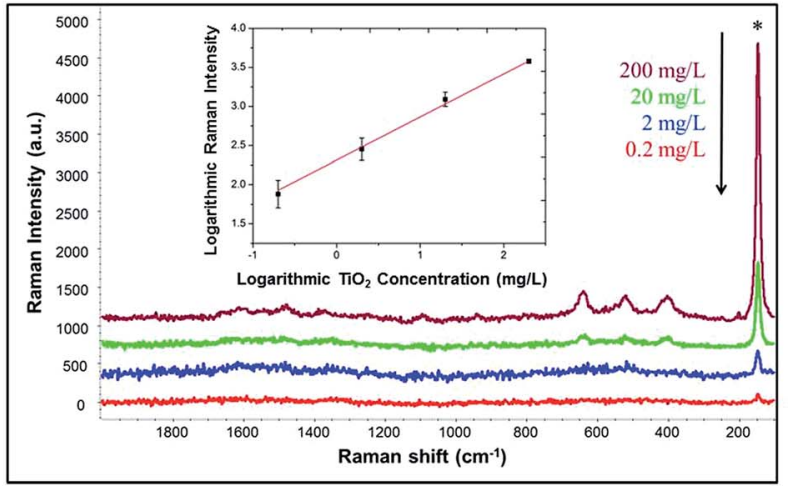

Fig. 5 (A) SERS spectra of different concentrations of $\mathrm{TiO}_{2} \mathrm{NPs}$ with (blue curves) and without extraction (red curves). $\mathrm{No} \mathrm{TiO}_{2} \mathrm{NPs} \mathrm{control}$ $\left(0 \mathrm{mg} \mathrm{L}^{-1}\right)$ is also shown (purple curve). (B) SERS spectra of extracted $\mathrm{TiO}_{2}$ NPs using MYC-assisted extraction method. Inset: linear fitting curve for quantification of extracted $\mathrm{TiO}_{2} \mathrm{NPs}$ based on $144 \mathrm{~cm}^{-1}$ Raman peak.

In all cases (Fig. 6), SERS spectra showed characteristic peaks of both MYC and $\mathrm{TiO}_{2}$ NPs at similar intensities to the controls (extraction tests under $\mathrm{pH} 7$ in the absence of interferences, Fig. 6B, pH 7). The results demonstrate that our developed method is fit for purpose with regard to the detection and quantification of $\mathrm{TiO}_{2}$ NPs, including in relatively complex sample matrices.

We verified the extraction efficiency of flavonoid-assisted extraction for $\mathrm{TiO}_{2}$ NPs by ICP-MS. As shown in Fig. 7, the recoveries of $68.9 \%, 88.3 \%, 81.2 \%$, and $87.1 \%$ were achieved at $0.2 \mathrm{mg} \mathrm{L}^{-1}, 2 \mathrm{mg} \mathrm{L}^{-1}, 20 \mathrm{mg} \mathrm{L}^{-1}$ and $200 \mathrm{mg} \mathrm{L}^{-1} \mathrm{TiO}_{2} \mathrm{NPs}$, respectively, verifying the suitability of the current method for

Table 2 Assessment of the assay's accuracy for aqueous samples $(n=5)$

Nominal

$\left[\mathrm{TiO}_{2}\right]\left(\mathrm{mg} \mathrm{L}^{-1}\right)$

Mean\% RV

0.2

2

20

200

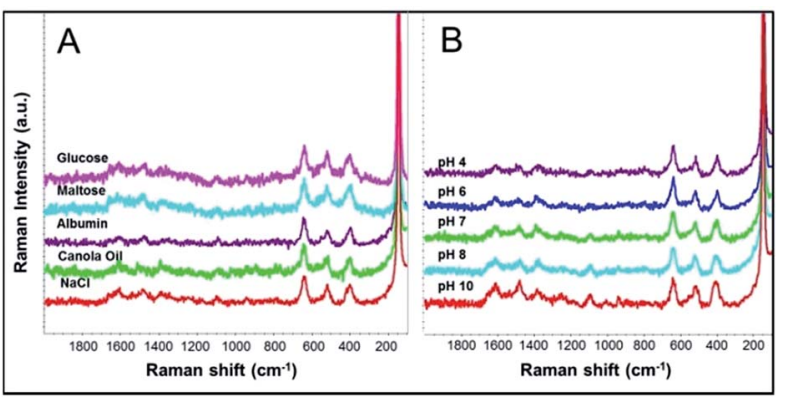

Fig. 6 Effects of complex matrices on MYC-assisted and SERS detection for $\mathrm{TiO}_{2} \mathrm{NPs}$ analysis, including various interferences (A) and $\mathrm{pH}$ conditions (B). The concentration of $\mathrm{TiO}_{2} \mathrm{NPs}$ for all cases is $200 \mathrm{mg} \mathrm{L}^{-1}$.

the high-efficiency extraction of these NPs at different concentrations. The effects of complex matrix including interferences and $\mathrm{pH}$ on extraction efficiency were also studied. For various interferences, the extraction efficiency of $200 \mathrm{mg} \mathrm{L}^{-1} \mathrm{TiO}_{2} \mathrm{NPs}$ in the presence of glucose, maltose, albumin, canola oil and $\mathrm{NaCl}$ were $71.1 \%, 83.5 \%, 84.2 \%, 66.6 \%$ and $72.9 \%$, respectively. The extraction efficiencies of $200 \mathrm{mg} \mathrm{L}^{-1} \mathrm{TiO}_{2} \mathrm{NPs}$ under $\mathrm{pH} 4$, pH 6, pH 7, pH 8 and pH 10 were $69.2 \%, 77.9 \%, 87.1 \%, 69.7 \%$ and $74.9 \%$, respectively. This data demonstrate the strong binding affinity of MYC to $\mathrm{TiO}_{2}$ even in the presence of many interferences and the good robustness of this method.

The overall extraction efficiency in this study (66.6-88.3\%) were satisfied and could compare favourably with the recoveries of $61.5 \%$ to $78.5 \%$ reported by a previous study using 11 -mercaptoundecanoic acid and octadecylamine as surfactant and cyclohexane as extraction solvent. ${ }^{10}$ In addition, centrifugation and evaporation were used to concentrate the extracted NPs in their study, which is time consuming. In another study, alkylphosphonic acids and chloroform were used for simultaneous surface modification and phase transfer of $\mathrm{TiO}_{2} \mathrm{NPs}^{33}$ Although they claimed total transfer by eye observation, overnight evaporating was used to concentrate the transferred $\mathrm{TiO}_{2} \mathrm{NPs}$, which is again time consuming. In contrast, our developed method is much

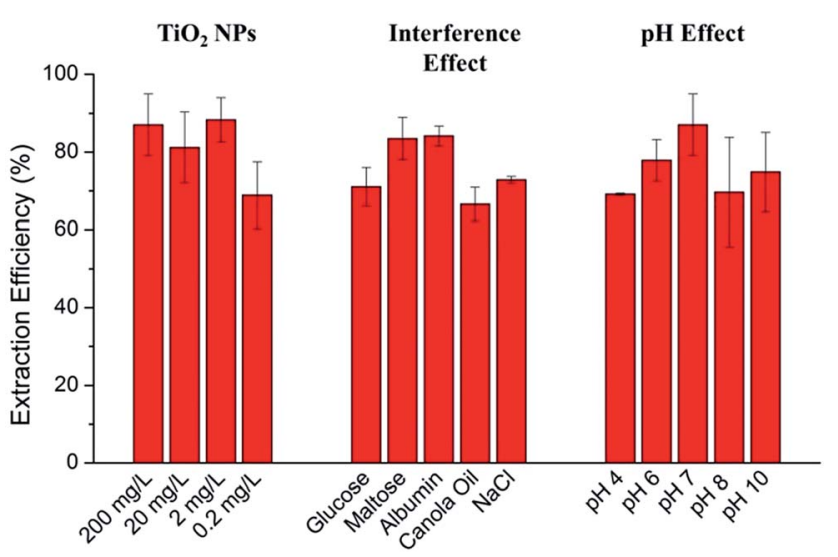

Fig. 7 Extraction efficiency of variable concentrations of $\mathrm{TiO}_{2} \mathrm{NPs}$ and effects of complex matrices (interferences and $\mathrm{pH}$ ) on extraction efficiency of $200 \mathrm{mg} \mathrm{L}^{-1} \mathrm{TiO}_{2} \mathrm{NPs}$.
$83.7 \pm 7.1$ $96.1 \pm 5.7$ $101.6 \pm 9.2$ $98.9 \pm 3.5$ 
faster $(<1 \mathrm{~h})$ and more convenient due to the fact that $\mathrm{TiO}_{2} \mathrm{NPs}$ were extracted and concentrated into an interlayer between aqueous phase and organic phase. Furthermore, our method is considered green because of the use of non-toxic and naturally existed chemicals, including flavonoids, sodium chlorite, and ethyl acetate. Ethyl acetate is a natural compound found in a variety of food (e.g. wine) and considered as a green solvent in many studies and reports. ${ }^{34-37}$ For example, it is reported as a solvent to develop green methods for alkene epoxidation. ${ }^{34}$

\section{$\mathrm{TiO}_{2}$ NPs extraction from real food samples}

Finally, the developed flavonoid-assisted microextraction method was challenged with real food samples, milk powder. Infant milk powder (no detectable $\mathrm{TiO}_{2}$ ) artificially added with $\mathrm{TiO}_{2}$ NPs were used as standard samples for the preparation of milk powder dispersions. Under the consideration of developing simple analytical method for real samples detection and avoid additional complicated procedures, sample pretreatment was performed in terms of simple diluting step that is supremely suitable for on-site measurement. Interestingly, we found that the extraction method could work satisfactorily with 100 times milk powder dilutions, but not undiluted dispersions and 10 dilutions due to the matrix interference at high concentration (data not shown). ${ }^{38}$ A series of milk powder dilutions containing different concentrations of $\mathrm{TiO}_{2} \mathrm{NPs}$ were tested.

As shown in Fig. 8A, SERS spectra show characteristic peaks of both MYC and $\mathrm{TiO}_{2}$ NPs and the Raman signal intensity decreased as the concentration of $\mathrm{TiO}_{2}$ NPs decreased from $50 \mathrm{mg} \mathrm{L}{ }^{-1}$ to $0.5 \mathrm{mg} \mathrm{L}^{-1}$. In contrast, the sample containing $0 \mathrm{mg} \mathrm{L}{ }^{-1} \mathrm{TiO}_{2}$ NPs, namely no $\mathrm{TiO}_{2}$ NPs control, did not show any Raman signals. We can detect as low as $0.5 \mathrm{mg} \mathrm{L}{ }^{-1} \mathrm{TiO}_{2} \mathrm{NPS}$ from milk powder dilutions, corresponding to $100 \mathrm{mg} \mathrm{\textrm {L } ^ { - 1 }}$ ( $0.01 \%$ by weight) which is likewise much lower than the $1 \%$ (by weight) limit set for $\mathrm{TiO}_{2}$ in foods. Based on the characteristic peak of $\mathrm{TiO}_{2} \mathrm{NPs}$ at $144 \mathrm{~cm}^{-1}$, the extracted $\mathrm{TiO}_{2} \mathrm{NPs}$ from milk powder shows a good linear response in the concentration range from $0.5 \mathrm{mg} \mathrm{L}^{-1}$ to $50 \mathrm{mg} \mathrm{L}^{-1}$ (Fig. 8B). The linear fitting equation is $\log Y=0.58 \log X+2.30$ and $R^{2}=0.980$. We also
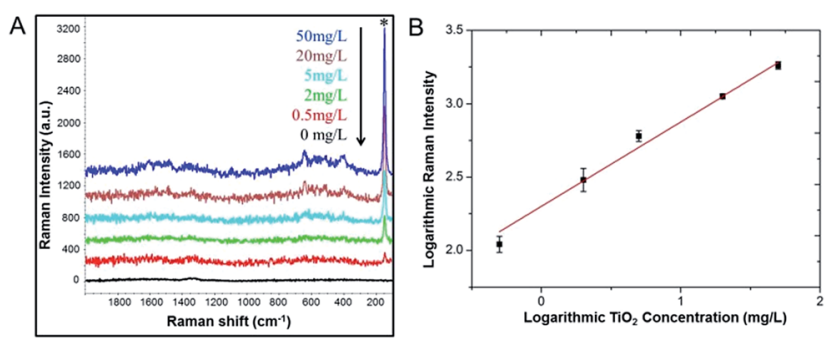

Fig. 8 (A) SERS spectra of extracted $\mathrm{TiO}_{2}$ NPs from milk powder dilutions using MYC-assisted extraction method. The milk powder suspension containing different concentrations of $\mathrm{TiO}_{2} \mathrm{NPs}$ was diluted by 100 times with water, followed by MYC-assisted microextraction. The final concentration of diluted $\mathrm{TiO}_{2} \mathrm{NPs}$ in extraction system was $50,20,5,2,0.5$ and $0 \mathrm{mg} \mathrm{L}^{-1}$, respectively. (B) Linear fitting curve for quantification of extracted $\mathrm{TiO}_{2} \mathrm{NPs}$ from milk powder dilutions based on $144 \mathrm{~cm}^{-1}$ Raman peak.
Table 3 Assessment of the assay's accuracy for real samples $(n=5)$

\begin{tabular}{lr}
$\begin{array}{l}\text { Nominal } \\
{\left[\mathrm{TiO}_{2}\right]\left(\mathrm{mg} \mathrm{L}^{-1}\right)}\end{array}$ & Mean\% RV \\
\hline 0.5 & $87.2 \pm 9.5$ \\
5 & $98.6 \pm 8.3$ \\
50 & $103.7 \pm 5.5$
\end{tabular}

evaluated the accuracy of the assay for powder milk dilutions containing different concentrations of $\mathrm{TiO}_{2} \mathrm{NPs}$ by calculating $\%$ RV based on regression equation (Table 3 ). The mean \% RV for $\mathrm{TiO}_{2}$ assay level ranged from 87.2 to $103.7 \%$, suggesting that the assay is able to accurately analyze $\mathrm{TiO}_{2}$ NPs in real samples. The results demonstrated the good capability and accuracy of the developed method for the extraction and quantification of $\mathrm{TiO}_{2}$ NPs from milk powder samples.

\section{Conclusions}

In conclusion, we present a novel method using flavonoidassisted extraction and Raman detection for $\mathrm{TiO}_{2}$ NPs analysis in real food samples. Using MYC, we were able to extract and detect concentration as low as $0.5 \mathrm{mg} \mathrm{L}{ }^{-1} \mathrm{TiO}_{2}$ NPs from milk powder dilutions. The developed method was sufficiently robust, performing well in various complex matrices and at a range of $\mathrm{pH}$ values. Although the sensitivity of the developed Raman detection method is not as great as ICP-MS, the developed method has several advantages. First, the use of non-toxic flavonoids, ethyl acetate, and sodium chloride could significantly decrease safety concerns caused by hazardous chemical use, especially hydrofluoric acid. Second, the method is both facile (no centrifugation needed) and rapid (analysis time $<1 \mathrm{~h}$ ), and has significant potential for on-site or field detection with a portable Raman instrument. Future work will focus on the optimization of this method to improve the sensitivity as well as study $\mathrm{TiO}_{2}$ of different types and particle sizes. The feasibility of using a portable Raman instrument for on-site measurement of $\mathrm{TiO}_{2}$ NPs will be explored.

\section{Acknowledgements}

We acknowledge the National Institute of Food and Agriculture of the U.S. Department of Agriculture (USDA-NIFA, grant no. 2015-67017-23070) for partially supporting this work.

\section{Notes and references}

1 X. Chen and S. S. Mao, Chem. Rev., 2007, 107, 2891-2959.

2 A. Weir, P. Westerhoff, L. Fabricius, K. Hristovski and N. von Goetz, Environ. Sci. Technol., 2012, 46, 2242-2250.

3 C. Chawengkijwanich and Y. Hayata, Int. J. Food Microbiol., 2008, 123, 288-292.

4 M. Skocaj, M. Filipic, J. Petkovic and S. Novak, Radiol. Oncol., 2011, 45, 227-247.

5 B. Jovanović, Integr. Environ. Assess. Manage., 2015, 11, 10-20. 
6 V. K. Sharma, J. Environ. Sci. Health, Part A: Toxic/Hazard. Subst. Environ. Eng., 2009, 44, 1485-1495.

7 B. Trouiller, R. Reliene, A. Westbrook, P. Solaimani and R. H. Schiestl, Cancer Res., 2009, 69, 8784-8789.

8 C. M. Sayes, R. Wahi, P. A. Kurian, Y. Liu, J. L. West, K. D. Ausman, D. B. Warheit and V. L. Colvin, Toxicol. Sci., 2006, 92, 174-185.

9 G. Singh, C. Stephan, P. Westerhoff, D. Carlander and T. V. Duncan, Compr. Rev. Food Sci. Food Saf., 2014, 13, 693-704.

10 J. P. F. G. Helsper, R. J. B. Peters, M. E. M. van Bemmel, Z. E. H. Rivera, S. Wagner, F. von der Kammer, P. C. Tromp, T. Hofmann and S. Weigel, Anal. Bioanal. Chem., 2016, 408, 6679-6691.

11 R. J. B. Peters, G. van Bemmel, Z. Herrera-Rivera, H. P. F. G. Helsper, H. J. P. Marvin, S. Weigel, P. C. Tromp, A. G. Oomen, A. G. Rietveld and H. Bouwmeester, J. Agric. Food Chem., 2014, 62, 6285-6293.

12 S. M. Majedi, B. C. Kelly and H. K. Lee, Anal. Chim. Acta, 2013, 789, 47-57.

13 S. Kumar and A. K. Pandey, Sci. World J., 2013, 2013, 162750. 14 C. A. Rice-Evans, N. J. Miller and G. Paganga, Free Radical Biol. Med., 1996, 20, 933-956.

15 E. Tripoli, M. L. Guardia, S. Giammanco, D. D. Majo and M. Giammanco, Food Chem., 2007, 104, 466-479.

16 I. A. Janković, Z. V. Šaponjić, M. I. Čomor and J. M. Nedeljković, J. Phys. Chem. C, 2009, 113, 12645-12652.

17 I. A. Janković, Z. V. Šaponjić, E. S. Džunuzović and J. M. Nedeljković, Nanoscale Res. Lett., 2010, 5, 81-88.

18 X. Cao, C. Ma, Z. Gao, J. Zheng, L. He, D. J. McClements and H. Xiao, J. Agric. Food Chem., 2016, 64, 9436-9441.

19 S. Lee, D.-H. Seo, H.-L. Park, Y. Choi and S. Jung, Antonie Van Leeuwenhoek, 2003, 84, 201-207.

20 J. Kurepa, R. Nakabayashi, T. Paunesku, M. Suzuki, K. Saito, G. E. Woloschak and J. A. Smalle, Plant J., 2014, 77, 443-453.

21 L. Yang, X. Jiang, W. Ruan, B. Zhao, W. Xu and J. R. Lombardi, J. Phys. Chem. C, 2008, 112, 20095-20098.

22 Y. Zhang, A. H. Yuwono, J. Li and J. Wang, Micropor. Mesopor. Mat., 2008, 110, 242-249.
23 L. Yang, X. Jiang, W. Ruan, J. Yang, B. Zhao, W. Xu and J. R. Lombardi, J. Phys. Chem. C, 2009, 113, 16226-16231.

24 A. Musumeci, D. Gosztola, T. Schiller, N. M. Dimitrijevic, V. Mujica, D. Martin and T. Rajh, J. Am. Chem. Soc., 2009, 131, 6040-6041.

25 L. Yang, X. Jiang, W. Ruan, B. Zhao, W. Xu and J. R. Lombardi, J. Raman Spectrosc., 2009, 40, 2004-2008.

26 D. Maznichenko, K. Venkatakrishnan and B. Tan, J. Phys. Chem. C, 2013, 117, 578-583.

27 X. Zhang, Z. Yu, W. Ji, H. Sui, Q. Cong, X. Wang and B. Zhao, J. Phys. Chem. C, 2015, 119, 22439-22444.

28 K. Colwell, Potentially hazardous nanoparticles found in powdered baby formula, http://www.foe.org/news/newsreleases/2016-05-potentially-hazardous-nanoparticlesfound-in-powdered-baby-formula, accessed 17.05, 2016.

29 H. Guo, Z. Zhang, B. Xing, A. Mukherjee, C. Musante, J. C. White and L. He, Environ. Sci. Technol., 2015, 49, 4317-4324.

30 T. Yang, Z. Zhang, B. Zhao, R. Hou, A. Kinchla, J. M. Clark and L. He, Anal. Chem., 2016, 88, 5243-5250.

31 R. A. French, A. R. Jacobson, B. Kim, S. L. Isley, R. L. Penn and P. C. Baveye, Environ. Sci. Technol., 2009, 43, 1354-1359.

32 U. S. Environmental Protection Agency, Field Portable X-ray Fluorescence for the Determination of Elemental Concentrations in Soil and Sediment, 1998, Method 6200.

33 C. Schmitt Pauly, A.-C. Genix, J. G. Alauzun, G. Guerrero, M.-S. Appavou, J. Pérez, J. Oberdisse and P. H. Mutin, Langmuir, 2015, 31, 10966-10974.

34 Y. Ding, W. Zhao, H. Hua and B. Ma, Green Chem., 2008, 10, 910-913.

35 E. G. Ankudey, H. F. Olivo and T. L. Peeples, Green Chem., 2006, 8, 923-926.

36 P. G. Jessop, Green Chem., 2011, 13, 1391-1398.

37 C. Capello, U. Fischer and K. Hungerbuhler, Green Chem., 2007, 9, 927-934.

38 N. Bordenave, B. R. Hamaker and M. G. Ferruzzi, Food Funct., 2014, 5, 18-34. 